\title{
GLOBAL ASYMPTOTIC STABILITY OF NONLINEAR PERIODIC IMPULSIVE EQUATIONS
}

\author{
V.I. SLYN'KO AND CEMIL TUNÇ
}

Received 01 December, 2017

\begin{abstract}
Pseudo-linear impulsive differential equations in a Banach space are considered. It is assumed that the conditions of a small change in the operator coefficients of the equation are satisfied. Using the method of "frozen" coefficients and the methods of commutator calculus, the problem of global asymptotic stability of a pseudo-linear impulsive differential equation is reduced to the problem of estimating the evolution operator for linear impulsive differential equation with constant operator coefficients. The obtained results are applied for stability study of a nonlinear system of ordinary impulsive differential equations. Lyapunov's direct method is used for estimating the fundamental matrix of the corresponding system of impulsive differential equations with constant coefficients. The stability conditions are formulated in terms of the solvability of certain linear matrix inequalities.
\end{abstract}

2010 Mathematics Subject Classification: 34D23; 34G20; 34K45

Keywords: commutator calculus, Lyapunov's direct method, Baker-Campbell-Hausdorff formula, impulsive systems, global stability, Magnus series

The stability of solutions of the impulsive differential equations has been studied in many papers $[3,4,7,8,11]$. In the monographs, [7, 11], the direct Lyapunov's method for nonlinear systems of impulsive differential equations is developed, and some results related to the first method of Lyapunov are presented.

In [8], the direct Lyapunov's method is developed using the second derivatives of the auxiliary function, while in [3] the higher derivatives of the Lyapunov function are used.

The problems of the global existence of solutions and the stability of abstract differential equations were also considered in the papers [13].

An actual and little studied problem of the theory of stability for solutions of impulsive differential equations is to find or to estimate the stability region, in particular, the establishment of the conditions for global asymptotic stability of the equilibrium position for this class of systems. Some results in this direction have been obtained

The first author was partially supported by the Ministry of Education and Science of Ukraine project No. 0116 U004691. 
in [2] for pseudolinear impulsive systems, under additional monotonicity conditions for these systems with respect to a certain cone in the phase space.

In the present paper, based on the methods of "frozen" coefficients [10] and the direct Lyapunov's method, in combination with the methods of commutator calculus, the problem of global stability of the zero solution of a nonlinear periodic impulsive differential equation in Banach space is investigated.

\section{AuXiliary RESUlts}

In this section, we give some basic algebraic concepts that will be used later (See [9]). Let us describe the construction of the free associative algebra $\mathfrak{R}$ over the field of real numbers $\mathbb{R}$ with two generators $x, y$. A word over the alphabet $(x, y)$ is a finite sequence of symbols from the alphabet, and for the sequence $\underbrace{z \ldots z}_{n}$, where $z$ is the generator, we use the notation $z^{n}, n \in \mathbb{N}, z^{0}=1$. The basis of the algebra $\mathfrak{R}_{0}$ consists of all words over the alphabet $(x, y)$. The empty word is identified with the element $1 \in \mathbb{R}$, therefore $\mathbb{R} \subset \mathfrak{R}_{0}$ and the field $\mathbb{R}$ is the center of algebra $\mathfrak{R}_{0}$. Consequently, $\mathfrak{R}_{0}$ consists of all possible linear combinations of words with coefficients from $\mathbb{R}$. Free associative algebra $\mathfrak{R}$ is defined as the completion of the algebra $\mathfrak{R}_{0}$ in some special topology and consists of all formal infinite series with coefficients from the field $\mathbb{R}$. Commutator of two elements $a \in \mathfrak{R}, b \in \mathfrak{R}$ which is defined by the formula

$$
[a, b]=a b-b a
$$

introduces into $\mathfrak{R}$ the structure of the Lie algebra. The commutation operator ad ${ }_{a}$, $a \in \mathfrak{R}$ is defined as a linear mapping $\mathfrak{R} \rightarrow \mathfrak{R}$,

$$
\operatorname{ad}_{a}(y)=[a, y], \quad y \in \Re .
$$

Let $f(x, y) \in \mathfrak{R}, z \in \mathfrak{R}, \lambda \in \mathbb{R}$. Then the polarization identity

$$
f(x+\lambda z, y)=f(x, y)+\lambda f_{1}(x, y, z)+\lambda^{2} f_{2}(x, y, z)+\ldots
$$

defines the derivative of Hausdorff $\left(z \frac{\partial}{\partial x}\right) f \stackrel{d f}{=} f_{1}(x, y, z)$.

We define recursively the following Lie elements of the algebra $\mathfrak{R}$

$$
\left\{y, x^{0}\right\}=y, \quad\left\{y, x^{l+1}\right\}=\left[\left\{y, x^{l}\right\}, x\right], \quad l \in \mathbb{N} .
$$

It is easy to see that

$$
\operatorname{ad}_{x}^{l}(y)=(-1)^{l}\left\{y, x^{l}\right\} .
$$

If $p(x)=\sum_{k=0}^{\infty} p_{k} x^{k}$ is a series from the generator $x$, then

$$
\{y, p(x)\} \stackrel{d f}{=} \sum_{k=0}^{\infty} p_{k}\left\{y, x^{k}\right\} .
$$


We also note that if $p(x)=\sum_{k=0}^{\infty} p_{k} x^{k}, q(x)=\sum_{k=0}^{\infty} q_{k} x^{k}$, then

$$
\{y, p(x) q(x)\}=\{\{y, p(x)\}, q(x)\} .
$$

Similarly, for an arbitrary finite sequence $x_{1}, \ldots, x_{n}$ of the elements from $\Re$, we define recursively an element $\left\{x_{1}, \ldots, x_{n-1}, x_{n}\right\}$ as

$$
\left\{x_{1}, \ldots, x_{n-1}, x_{n}\right\}=\left[\left\{x_{1}, \ldots, x_{n-1}\right\}, x_{n}\right] .
$$

Moreover, we have

$$
e^{-x} y e^{x}=\left\{y, e^{x}\right\}
$$

and the following identities of F. Hausdorff are valid

$$
e^{-x}\left(y \frac{\partial}{\partial x}\right) e^{x}=\left\{y, \frac{e^{x}-1}{x}\right\}, \quad\left(\left(y \frac{\partial}{\partial x}\right) e^{x}\right) e^{-x}=\left\{y, \frac{1-e^{-x}}{x}\right\} .
$$

Note that the identities (1.1) - (1.3) have a formal character.

If $X$ is a Banach space and $L(X)$ is a Banach algebra of bounded linear operators acting in $X$, then (1.1) is satisfied in the common part of the domain of convergence of the series $p(x), q(x)$, and (1.2), (1.3) are satisfied for all $x \in L(X), y \in L(X)$.

Consider the operator differential equation

$$
\frac{d U(t, s)}{d t}=A(t) U(t, s), \quad U(s, s)=1, \quad t \geq s,
$$

where $U \in C^{1}(\mathbb{R} \times \mathbb{R} ; L(X)), A \in C(\mathbb{R} ; L(X))$.

In paper [9] W. Magnus has established the conditions under which the operator $U(t)$ can be represented as

$$
U(t, s)=\exp \hat{\Omega}(t, s), \quad t \geq s .
$$

In addition, the function $\hat{\Omega}(t, s)$ is a solution of the Cauchy problem for a nonlinear differential equation (W. Magnus equation)

$$
\frac{d \hat{\Omega}(t, s)}{d t}=\left\{A(t), \frac{\hat{\Omega}(t, s)}{1-e^{-\hat{\Omega}(t, s)}}\right\}=\sum_{n=0}^{\infty} \beta_{n}\left\{A(t), \hat{\Omega}^{n}(t, s)\right\}, \quad \hat{\Omega}(s, s)=0 .
$$

Here $\beta_{1}=1 / 2, \beta_{n}=0$ for $n=3,5, \ldots, \beta_{2 n}=\frac{(-1)^{n-1} B_{2 n}}{(2 n) !}$, for $n \in \mathbb{N}$ and $\beta_{0}=1$, $B_{2 n}$ are Bernoulli numbers [5].

Integration of this Cauchy problem (1.5) leads to the formal series of W. Magnus. This series is a continual generalization of the classical Baker-Campbell-Hausdorff formula. It is well known that the series on the right-hand side of this formula does not always converge. Therefore, in the present paper, we will use the following representation of the solution of the Cauchy problem (1.4)

$$
U(t, s)=(I+F(t, s)) e^{\int_{s}^{t} A(\tau) d \tau},
$$

where $F(t, s)$ is the function defined below, $I$ is identity operator. 
Next we will derive a differential operator equation for the function $F(t, s)$. Applying the chain rule for the derivative of a composite function [9], we obtain

$$
\frac{d F(t, s)}{d t}=\frac{d U(t, s)}{d t} e^{-\hat{A}(t, s)}-\left.U(t, s)\left(A(t) \frac{\partial}{\partial X}\right) e^{X}\right|_{X=-\hat{A}(t, s)},
$$

where $\hat{A}(t, s)=\int_{s}^{t} A(\tau) d \tau$.

Hence, taking into account the Hausdorff identity, we get

$$
\begin{aligned}
& \frac{d F(t, s)}{d t}=A(t) U(t, s) e^{-\hat{A}(t, s)}+U(t, s) e^{-\int_{s}^{t} A(\tau) d \tau}\left\{A(t), \frac{e^{-\hat{A}(t, s)}-1}{\hat{A}(t, s)}\right\} \\
&=A(t)(F(t, s)+I)-(F(t, s)+I)(A(t)-\Psi(t, s)) \\
&=[A(t), F(t, s)]+F(t, s) \Psi(t, s)+\Psi(t, s),
\end{aligned}
$$

where

$$
\Psi(t, s)=\sum_{k=1}^{\infty} \frac{(-1)^{k+1}}{(k+1) !}\left\{A(t), \hat{A}^{k}(t, s)\right\} .
$$

Thus, the function $F(t, s)$ is a solution of the Cauchy problem

$$
\frac{d F(t, s)}{d t}=[A(t), F(t, s)]+F(t, s) \Psi(t, s)+\Psi(t, s), \quad F(s, s)=0 .
$$

For further discussion, it is necessary to recall the notion of the logarithmic operator norm [1]. For a bounded linear operator $A \in L(X)$, the logarithmic operator norm $\Lambda(A)$ is defined as follows

$$
\Lambda(A)=\lim _{\varepsilon \rightarrow 0+} \frac{\|I+\varepsilon A\|-1}{\varepsilon},
$$

where $\varepsilon$ is real numbers.

\section{Problem statement}

Consider the impulsive differential equation

$$
\begin{gathered}
\frac{d x(t)}{d t}=A(t, x(t)) x(t), \quad t \neq n \theta, \\
\Delta x(t)=B(x(t)) x(t), \quad t=n \theta,
\end{gathered}
$$

where $x \in X, A \in C^{0,1}(\mathbb{R} \times X ; L(X)), \theta>0, \Delta x(t)=x(t+0)-x(t), x(t+0)=$ $\lim _{s \rightarrow t, s>t} x(s), B \in C^{1}(X ; L(X))$ and $A(t, x)$ is a $\theta$-periodic function of $t$.

Let $x\left(t ; x_{0}\right)$ be a solution of the Cauchy problem (2.1) with initial condition $x(0+$ $\left.0, x_{0}\right)=x_{0}$. In this case, as usual, it is assumed that $x\left(t-0 ; x_{0}\right)=x\left(t, x_{0}\right)$.

The aim of this paper is to obtain sufficient conditions for the global asymptotic stability of the solution $x=0$ of the impulsive differential equation (2.1). 
Definition 1. The solution $x=0$ of (2.1) is said to be globally asymptotically stable if for any positive number $\varepsilon$ there exists a positive number $\delta=\delta(\varepsilon)$ such that the inequality $\left\|x_{0}\right\|<\delta$ implies the estimate $\left\|x\left(t ; x_{0}\right)\right\|<\varepsilon$ for all $t \geq 0$ and for all $x_{0} \in X$

$$
\lim _{t \rightarrow \infty}\left\|x\left(t, x_{0}\right)\right\|=0 .
$$

We will study the global asymptotic stability of the solution $x=0$ under the following additional assumptions.

Assumption 1. We assume that for the differential equation (2.1) the following conditions are satisfied:

(1) There exist positive constants $a$ and $b$ such that for all $(t, x) \in \mathbb{R} \times X$ the inequalities

$$
\sup _{(t, x) \in[0, \theta] \times X}\|A(t, x)\| \leq a, \quad \sup _{(t, x) \in[0, \theta] \times X}\left\|A_{x}^{\prime}(t, x)\right\| \leq b
$$

are fulfilled, where $A_{x}^{\prime} \in L(X, L(X))$ is a Frechet derivative of $x$ [6].

(2) There exist functions $\alpha \in C([0, \theta] ; \mathbb{R}), \gamma \in C\left([0, \theta] \times[0, \theta] ; \mathbb{R}_{+}\right)$such that for all $t \in[0, \theta]$ the following inequalities hold

$$
\sup _{x \in X} \Lambda(A(t, x)) \leq \alpha(t), \quad \sup _{x \in X}\left\|A_{x}^{\prime}(t, x)(A(\tau, x) x)\right\| \leq \gamma(t, \tau) .
$$

(3) There exists function $\beta \in C\left(\mathbb{R} ; \mathbb{R}_{+}\right)$such that for all $t \in[0, \theta]$ the following estimate is valid

$$
\sup _{x \in X}\left\|B_{x}^{\prime}(x)(A(t, x) x)\right\| \leq \beta(t) .
$$

\section{MAin RESUlt}

Consider the nonlinear impulsive differential equation (2.1). Let $T \in(0, \theta], t_{k}=$ $k h, k=0,1, \ldots, s, h=\frac{T}{s}$. We define the function $x_{h}\left(t ; x_{0}\right)$ inductively by the formulas

$$
x_{h}\left(0 ; x_{0}\right)=x_{0}, \quad x_{h}\left(t ; x_{0}\right)=e^{(t-k h) A\left(t_{k}, x_{k}\right)} x_{k}, \quad t \in(k h,(k+1) h],
$$

where $x_{k}=x_{h}\left(k h ; x_{0}\right)$.

Using the continuity of the operator-valued function $A(t, x)$ and the integral inequality of Gronwall-Bellman, we can prove the validity of the following auxiliary assertions (Lemma 1 and Lemma 2), similar to how it was done in [12].

Lemma 1. Assume that for the impulsive differential equation (2.1) the condition (1) of Assumption 1 holds. Then uniformly over $t \in[0, \theta]$, we get

$$
\lim _{h \rightarrow 0+}\left\|x\left(t ; x_{0}\right)-x_{h}\left(t ; x_{0}\right)\right\|=0 .
$$


Along with the impulsive differential equation (2.1), we consider the linear impulsive differential equation

$$
\begin{aligned}
& \frac{d y(t)}{d t}=A\left(t, x_{0}\right) y(t), \quad t \neq n \theta, \quad y(0+0)=x_{0}, \\
& \Delta y(t)=B\left(x_{0}\right) y(t), \quad t=n \theta,
\end{aligned}
$$

where $y(t) \in X, x_{0} \in X$. Similarly, we define a continuous function for $t \in(k h,(k+$ 1) $h$ ] by the formula

$$
y_{h}\left(t, x_{0}\right)=e^{A\left(k h, x_{0}\right)(t-k h)} e^{A\left((k-1) h, x_{0}\right) h} \ldots e^{A\left(0, x_{0}\right) h} x_{0} .
$$

Similarly, we can prove the following statement.

Lemma 2. Assume that for the impulsive differential equation (2.1) the condition (1) of Assumption 1 holds. Then uniformly over $t \in(0, \theta]$ we have

$$
\lim _{h \rightarrow 0+}\left\|y\left(t ; x_{0}\right)-y_{h}\left(t ; x_{0}\right)\right\|_{X}=0 .
$$

The following statement establishes an estimate of the error in the "freezing" of the coefficients of the equation (2.1) and is a generalization of Lemma 5.3 from the monograph [12], in which the autonomous case is considered.

Lemma 3 (cf. [2]). If the conditions of Assumption 1 are satisfied, then the following inequalities hold

$$
\begin{gathered}
\left\|x\left(\theta ; x_{0}\right)-y\left(\theta ; x_{0}\right)\right\| \leq e^{\int_{0}^{\theta} \alpha(s) d s}\left(e^{\iint_{0}^{\theta s} \gamma(s, \eta) d s d \eta}-1\right)\left\|x_{0}\right\|, \\
\left\|B\left(x\left(\theta ; x_{0}\right)\right)-B\left(x_{0}\right)\right\| \leq \int_{0}^{\theta} \beta(s) d s .
\end{gathered}
$$

Along with the original impulsive differential equation (2.1), we consider the linear differential equation

$$
\begin{aligned}
\frac{d z(t)}{d t} & =A_{0}\left(x_{0}\right) z(t), \quad t \neq n \theta, \\
\Delta z(t) & =\left(B\left(x_{0}\right)+\hat{B}\left(x_{0}\right)+B\left(x_{0}\right) \hat{B}\left(x_{0}\right)\right) z(t), \quad t=n \theta,
\end{aligned}
$$

where $z \in X$, and the linear operators $A_{0}\left(x_{0}\right)$ and $\hat{B}_{0}\left(x_{0}\right)$ are defined as follows

$$
\begin{aligned}
& A_{0}\left(x_{0}\right)=\frac{1}{\theta} \int_{0}^{\theta} A\left(\tau, x_{0}\right) d \tau, \quad \hat{A}\left(t, x_{0}\right)=\int_{0}^{t} A\left(\tau, x_{0}\right) d \tau, \quad \hat{B}\left(x_{0}\right)=F\left(\theta, x_{0}\right), \\
& \frac{d F\left(t, x_{0}\right)}{d t}=\left[A\left(t, x_{0}\right), F\left(t, x_{0}\right)\right]+F\left(t, x_{0}\right) \Psi\left(t, x_{0}\right)+\Psi\left(t, x_{0}\right), \quad F\left(0, x_{0}\right)=0,
\end{aligned}
$$


where

$$
\Psi\left(t, x_{0}\right)=\sum_{k=1}^{\infty} \frac{(-1)^{k+1}}{(k+1) !}\left\{A\left(t, x_{0}\right), \hat{A}^{k}\left(t, x_{0}\right)\right\} .
$$

Define a monodromy operator of the linear impulsive differential equation (3.2) by

$$
U\left(x_{0}\right)=\left(I+B\left(x_{0}\right)\right)\left(I+\hat{B}\left(x_{0}\right)\right) e^{\theta A_{0}\left(x_{0}\right)} .
$$

The following statement is the main result of this paper.

Theorem 1. Assume that the conditions of Assumption 1 are valid for impulsive differential equation (2.1) and there exist positive constants $\zeta_{i}, i=1,2,3$ such that

$$
\sup _{x_{0} \in X}\left\|U\left(x_{0}\right)\right\| \leq \zeta_{1}, \quad \sup _{x_{0} \in X}\left\|\left(I+\hat{B}\left(x_{0}\right)\right) e^{\theta A_{0}\left(x_{0}\right)}\right\| \leq \zeta_{2}, \quad \sup _{x_{0} \in X}\left\|I+B\left(x_{0}\right)\right\| \leq \zeta_{3} .
$$

Let

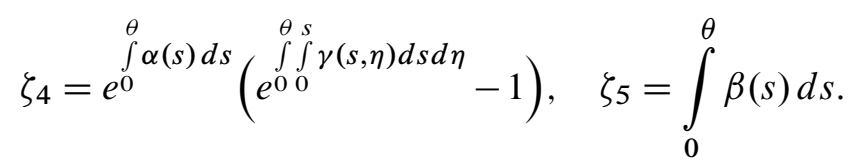

Then the fulfillment of the inequality

$$
\zeta_{1}+\zeta_{4} \zeta_{3}+\zeta_{5} \zeta_{2}+\zeta_{5} \zeta_{4}<1
$$

guarantees the global asymptotic stability of the solution $x=0$ of the differential equation (2.1).

Proof. We obtain

$$
\begin{aligned}
& x\left(\theta+0 ; x_{0}\right)-y\left(\theta+0 ; x_{0}\right)=\left(I+B\left(x_{0}\right)\right)\left(x\left(\theta ; x_{0}\right)-y\left(\theta ; x_{0}\right)\right) \\
& +\left(B\left(x\left(\theta ; x_{0}\right)\right)-B\left(x_{0}\right)\right) y\left(\theta ; x_{0}\right)+\left(B\left(x\left(\theta ; x_{0}\right)\right)-B\left(x_{0}\right)\right)\left(x\left(\theta ; x_{0}\right)-y\left(\theta ; x_{0}\right)\right) .
\end{aligned}
$$

From the assertion of Lemma 1 and the conditions of Theorem 1, it follows that

$$
\begin{aligned}
&\left\|x\left(\theta+0 ; x_{0}\right)-y\left(\theta+0 ; x_{0}\right)\right\| \leq\left\|I+B\left(x_{0}\right)\right\|\left\|x\left(\theta ; x_{0}\right)-y\left(\theta ; x_{0}\right)\right\| \\
&+\left\|\left(B\left(x\left(\theta ; x_{0}\right)\right)-B\left(x_{0}\right)\right) y\left(\theta ; x_{0}\right)\right\|+\| B\left(x\left(\theta ; x_{0}\right)\right)-B\left(x_{0}\right)\|\| x\left(\theta ; x_{0}\right)-y\left(\theta ; x_{0}\right) \| \\
& \leq \zeta_{4} \zeta_{3}\left\|x_{0}\right\|+\zeta_{5}\left\|y\left(\theta ; x_{0}\right)\right\|+\zeta_{5} \zeta_{4}\left\|x_{0}\right\| .
\end{aligned}
$$

Consider that

$$
\begin{aligned}
y\left(\theta ; x_{0}\right) & =\left(I+\hat{B}\left(x_{0}\right)\right) e^{\theta A_{0}\left(x_{0}\right)} x_{0}, \\
y\left(\theta+0 ; x_{0}\right) & =\left(I+B\left(x_{0}\right)\right)\left(I+\hat{B}\left(x_{0}\right)\right) e^{\theta A_{0}\left(x_{0}\right)} x_{0}=U\left(x_{0}\right) x_{0} .
\end{aligned}
$$

Then, we get

$$
\begin{aligned}
\left\|x\left(\theta+0 ; x_{0}\right)\right\| \leq\left\|U\left(x_{0}\right)\right\|\left\|x_{0}\right\|+ & \zeta_{4} \zeta_{3}\left\|x_{0}\right\|+\zeta_{5} \zeta_{2}\left\|x_{0}\right\|+\zeta_{5} \zeta_{4} \\
& \leq\left(\zeta_{1}+\zeta_{4} \zeta_{3}+\zeta_{5} \zeta_{2}+\zeta_{5} \zeta_{4}\right)\left\|x_{0}\right\|:=q\left\|x_{0}\right\| .
\end{aligned}
$$


Due to the periodicity of the differential equation (2.1), we obtain

$$
\left\|x\left(n \theta+0 ; x_{0}\right)\right\| \leq q^{n}\left\|x_{0}\right\|
$$

from which it follows the global asymptotic stability of the solution $x=0$ of the impulsive differential equation (2.1). Thus Theorem 1 is proved.

Thus, the study of the global stability of the solution $x=0$ for the nonlinear differential equation (2.1) is reduced to estimating the solutions of the linear differential equation with constant operator coefficients (3.2) and solving the auxiliary differential equation (3.3) to obtain the operator $\hat{B}\left(x_{0}\right)$. As well as for solving of the W. Magnus equation, one can use the method of successive approximations to solve this auxiliary equation.

Let us define recursively a sequence

$$
\begin{gathered}
F_{0}\left(t, x_{0}\right) \equiv 0, \\
F_{m+1}\left(t, x_{0}\right)=\int_{0}^{t}\left(\left[A\left(s, x_{0}\right), F_{m}\left(s, x_{0}\right)\right]+F_{m}\left(s, x_{0}\right) \Psi\left(s, x_{0}\right)+\Psi\left(s, x_{0}\right)\right) d s .
\end{gathered}
$$

Then, assuming that

$$
\begin{gathered}
\sup _{x_{0} \in X} \max _{s \in[0, \theta]}\left(\left\|\operatorname{ad}_{A\left(s, x_{0}\right)}\right\|+\left\|\Psi\left(s, x_{0}\right)\right\|\right) \leq \rho_{0}, \\
\sup _{x_{0} \in X} \max _{s \in[0, \theta]}\left\|\Psi\left(s, x_{0}\right)\right\| \leq \rho_{1},
\end{gathered}
$$

where $\rho_{i}, i=1,2$ are positive constants, we obtain

$$
\left\|F\left(t, x_{0}\right)-F_{m}\left(t, x_{0}\right)\right\| \leq \epsilon_{m} \rho_{1} \theta,
$$

where $\epsilon_{m}=e^{\theta \rho_{0}}-\sum_{k=0}^{m-1} \frac{\left(\theta \rho_{0}\right)^{k}}{k !}$.

Consider a linear impulsive differential equation of the form

$$
\begin{aligned}
\frac{d z(t)}{d t} & =A_{0}\left(x_{0}\right) z(t), \quad t \neq n \theta, \\
\Delta z(t) & =\left(B\left(x_{0}\right)+\hat{B}_{m}\left(x_{0}\right)+B\left(x_{0}\right) \hat{B}_{m}\left(x_{0}\right)\right) z(t), \quad t=n \theta,
\end{aligned}
$$

where $\hat{B}_{m}\left(x_{0}\right)=F_{m}\left(\theta, x_{0}\right)$, and let $U_{m}\left(x_{0}\right)$ be the monodromy operator of this equation

$$
U_{m}\left(x_{0}\right)=\left(I+B\left(x_{0}\right)\right)\left(I+\hat{B}_{m}\left(x_{0}\right)\right) e^{\theta A_{0}\left(x_{0}\right)} .
$$

Theorem 1 implies the following statement.

Corollary 1. Assume that for the impulsive differential equation (2.1) the conditions of Assumption 1 are satisfied and there exist positive constants $\zeta_{i}^{\prime}, i=1,2$ such 
that

$$
\sup _{x_{0} \in X}\left\|U_{m}\left(x_{0}\right)\right\| \leq \zeta_{1}^{\prime}, \quad \sup _{x_{0} \in X}\left\|\left(I+\hat{B}_{m}\left(x_{0}\right)\right) e^{\theta A_{0}\left(x_{0}\right)}\right\| \leq \zeta_{2}^{\prime}
$$

and a constant $\alpha_{0}$ such that $\sup _{x_{0} \in X} \Lambda\left(A_{0}\left(x_{0}\right)\right) \leq \alpha_{0}$.

If

$$
\zeta_{1}^{\prime}+\zeta_{4} \zeta_{3}+\zeta_{5} \zeta_{2}^{\prime}+\zeta_{4} \zeta_{5}+\zeta_{6}\left(\zeta_{3}+\zeta_{5}\right) e^{\alpha_{0} \theta}<1,
$$

where $\zeta_{6}=\theta \rho_{1} \epsilon_{m}$, then the solution $x=0$ of the impulsive differential equation (2.1) is globally asymptotically stable.

\section{EXAMPLE}

Consider a system of impulsive differential equations

$$
\begin{aligned}
& \frac{d x(t)}{d t}=\psi(x(t)) A(t) x(t), \quad t \neq n \theta, \\
& \Delta x(t)=\psi(x(t)) B x(t), \quad t=n \theta,
\end{aligned}
$$

where $x \in \mathbb{R}^{m}, \psi \in C^{1}\left(\mathbb{R}^{m}, \mathbb{R}\right), A \in C\left(\mathbb{R} ; L\left(\mathbb{R}^{m}\right)\right), A(t+\theta)=A(t), B \in L\left(\mathbb{R}^{m}\right)$.

Assume that a nonlinear function $\psi(x)$ satisfies the following condition. There exist positive constants $\sigma, \psi_{m}, \psi_{M}$ such that

$$
0<\psi_{m} \leq \psi(x) \leq \psi_{M}<+\infty, \quad \sup _{x \in \mathbb{R}^{n}}\|\nabla \psi(x)\|\|x\| \psi(x) \leq \sigma .
$$

Denote

$$
A_{0}=\frac{1}{\theta} \int_{0}^{\theta} A(\tau) d \tau, \quad \hat{A}(t)=\int_{0}^{t} A(\tau) d \tau, \quad \Psi_{m}(t)=\left\{A(t), \hat{A}^{m}(t)\right\} .
$$

The corresponding linear differential equation with "frozen" coefficients has the form

$$
\begin{aligned}
& \frac{d y(t)}{d t}=\psi\left(x_{0}\right) A_{0} y(t), \quad t \neq n \theta, \\
& \Delta y(t)=\left(\psi\left(x_{0}\right) B+\hat{B}\left(x_{0}\right)+\psi\left(x_{0}\right) B \hat{B}\left(x_{0}\right)\right) y(t), \quad t=n \theta,
\end{aligned}
$$

where $y \in \mathbb{R}^{n}, \hat{B}\left(x_{0}\right)=F\left(\theta, x_{0}\right), F\left(t, x_{0}\right)$ is a solution of the Cauchy problem

$$
\begin{gathered}
\frac{d F\left(t, x_{0}\right)}{d t}=\psi\left(x_{0}\right)\left[A(t), F\left(t, x_{0}\right)\right]+F\left(t, x_{0}\right) \Psi\left(t, x_{0}\right)+\Psi\left(t, x_{0}\right), \quad F\left(0, x_{0}\right)=0, \\
\Psi\left(t, x_{0}\right)=\sum_{k=1}^{\infty} \frac{(-1)^{k+1} \psi^{k+1}\left(x_{0}\right)}{(k+1) !}\left\{A(t), \hat{A}^{k}(t)\right\} .
\end{gathered}
$$


We represent the solution of the Cauchy problem (4.4) in the integral form

$$
\begin{aligned}
F\left(t, x_{0}\right) & =\int_{0}^{t} \Phi(t, s)\left(\frac{\psi\left(x_{0}\right)}{2}\left[A^{T}(s)+A(s), F\left(s, x_{0}\right)\right]\right. \\
& \left.+F\left(s, x_{0}\right) \Psi\left(s, x_{0}\right)+\Psi\left(s, x_{0}\right)\right) \Phi^{T}(t, s) d s,
\end{aligned}
$$

where operator $\Phi(t, s)$ is a solution of the Cauchy problem

$$
\frac{d \Phi(t, s)}{d t}=\frac{1}{2}\left(A(t)-A^{T}(t)\right) \Phi(t, s), \quad \Phi(s, s)=I .
$$

Since $\|\Phi(t, s)\|=1$, then from (4.4) it follows the estimate

$$
\begin{gathered}
\left\|F\left(t, x_{0}\right)\right\| \\
\leq \int_{0}^{t}\left(\frac{\psi_{M}}{2}\left\|\operatorname{ad}_{A^{T}(s)+A(s)}\right\|+\left\|\Psi\left(s, x_{0}\right)\right\|\right)\left\|F\left(s, x_{0}\right)\right\| d s+\int_{0}^{\theta}\left\|\Psi\left(s, x_{0}\right)\right\| d s .
\end{gathered}
$$

Applying the integral Gronwall-Bellman inequality, we obtain the estimate

$$
\left\|F\left(\theta, x_{0}\right)\right\| \leq \int_{0}^{\theta}\left\|\Psi\left(s, x_{0}\right)\right\| d s \exp \left(\int_{0}^{\theta}\left(\frac{\psi_{M}}{2}\left\|\operatorname{ad}_{A^{T}(s)+A(s)}\right\|+\left\|\Psi\left(s, x_{0}\right)\right\|\right) d s\right) .
$$

Since

then we obtain

$$
\left\|\Psi\left(t, x_{0}\right)\right\| \leq \sum_{k=1}^{\infty} \frac{\left\|\Psi_{k}(t)\right\| \psi_{M}^{k+1}}{(k+1) !}
$$

$$
\begin{aligned}
\left\|\hat{B}\left(x_{0}\right)\right\| \leq & \int_{0}^{\theta} \sum_{k=1}^{\infty} \frac{\left\|\Psi_{k}(s)\right\| \psi_{M}^{k+1}}{(k+1) !} d s \\
& \times \exp \left(\int_{0}^{\theta}\left(\frac{\psi_{M}}{2}\left\|\operatorname{ad}_{A^{T}(s)+A(s)}\right\|+\sum_{k=1}^{\infty} \frac{\left\|\Psi_{k}(s)\right\| \psi_{M}^{k+1}}{(k+1) !}\right) d s\right):=\eta .
\end{aligned}
$$

Next, we consider the question of estimating the monodromy matrix of the system of equations (4.3). Assume the linear operator $A_{0}$ satisfies the Routh-Hurwitz conditions, i.e. $\max _{\lambda \in \sigma\left(A_{0}\right)} \operatorname{Re} \lambda<0$. Then, the Lyapunov matrix equation

$$
A_{0}^{T} X+X A_{0}=-Q,
$$

where $Q$ is a symmetric positive-definite linear matrix, has a unique solution $X-\mathrm{a}$ symmetric positive-definite operator. 
Consider the Lyapunov function $v(y)=y^{T} X y$. Then

$$
\left.\frac{d v}{d t}\right|_{(4.3)} \leq-\psi\left(x_{0}\right) y^{T} Q y \leq-\frac{\psi_{m} \lambda_{m}(Q)}{\lambda_{M}(X)} v(y(t)), \quad t \neq n \theta .
$$

Denote

$$
\begin{gathered}
\bar{\psi}=\frac{\psi_{m}+\psi_{M}}{2}, \quad R=-\left(B^{T} X+X B+\bar{\psi} B^{T} X B\right), \\
\eta_{0}=\frac{\psi_{M}-\psi_{m}}{2}\left\|B^{T} X+X B\right\|+\frac{\left(\psi_{M}-\psi_{m}\right)\left(3 \psi_{M}+\psi_{m}\right)}{4}\left\|B^{T} X B\right\|, \\
\eta_{1}=\|X-\bar{\psi} R\|+\eta_{0}, \quad \vartheta=\frac{\eta_{0}+\eta(2+\eta) \eta_{1}}{\lambda_{m}(X)} .
\end{gathered}
$$

Therefore for $t=n \theta$ we obtain

$$
v(y(t+0)) \leq(1-\bar{\psi} \Lambda(R, X)+\vartheta) v(y(t)),
$$

where

$$
\Lambda(R, X)= \begin{cases}\frac{\lambda_{m}(R)}{\lambda_{M}(X)}, & \lambda_{m}(R) \geq 0 \\ \frac{\lambda_{m}(R)}{\lambda_{m}(X)}, & \lambda_{m}(R)<0 .\end{cases}
$$

From (4.6) and (4.7) it follows that

$$
\left\|U\left(x_{0}\right)\right\| \leq \sqrt{\frac{(1-\bar{\psi} \Lambda(R, X)+\vartheta) \lambda_{M}(X)}{\lambda_{m}(X)}} e^{-\theta \frac{\psi m \lambda m(Q)}{2 \lambda_{M}(X)}}:=\zeta_{1} .
$$

Similarly, we obtain

$$
\left\|\left(I+\hat{B}\left(x_{0}\right)\right) e^{\psi\left(x_{0}\right) \theta A_{0}}\right\| \leq(1+\eta) \sqrt{\frac{\lambda_{M}(X)}{\lambda_{m}(X)}} e^{-\theta \frac{\psi m \lambda m(Q)}{2 \lambda M^{(X)}}}:=\zeta_{2} .
$$

Taking into account the assumption (4.2), we get

$$
\begin{gathered}
\zeta_{3}=\|I+\bar{\psi} B\|+\frac{\psi_{M}-\psi_{m}}{2}\|B\|, \\
\zeta_{4}=e^{\psi_{0}^{*} \int_{0}^{\theta} \Lambda(A(s)) d s}\left(e^{\sigma \iint_{0}^{\theta \tau}\|A(\tau)\|\|A(s)\| d \tau d s}-1\right), \quad \zeta_{5}=\sigma\|B\| \int_{0}^{\theta}\|A(s)\| d s,
\end{gathered}
$$

where

$$
\psi^{*}=\left\{\begin{array}{lll}
\psi_{M}, & \text { for } \quad & \int_{0}^{\theta} \Lambda(A(s)) d s>0, \\
& & { }_{0}^{\theta} \Lambda(A(s)) d s \leq 0 .
\end{array}\right.
$$

Theorem 1 implies the following statement. 
Proposition 1. Assume that the condition (4.2) is satisfied for the system (4.1) and $\max _{\lambda \in \sigma(A)} \operatorname{Re} \lambda<0$. If the following inequality is valid $\lambda \in \sigma\left(A_{0}\right)$

$$
\zeta_{1}+\zeta_{4} \zeta_{3}+\zeta_{5} \zeta_{2}+\zeta_{4} \zeta_{5}<1
$$

then the solution $x=0$ of the system (4.1) is globally asymptotically stable.

Next we will consider the case when, in general, the matrix $A_{0}$ does not satisfy the Routh-Hurwitz condition. Assume that for a given positive-definite symmetric matrix $G$ there exists a positive-definite matrix $X$ satisfying the conditions

$$
\begin{gathered}
\theta\left(A_{0}^{T} X+X A_{0}\right)+B^{T} X+X B+\bar{\psi} B^{T} X B=-G, \\
A_{0}^{T 2} X+2 A_{0}^{T} X A_{0}+X A_{0}^{2} \geq 0 .
\end{gathered}
$$

Consider the Lyapunov function $v(y)=y^{T} X y$. Then we get

$$
v\left(x_{0}\right)-v\left(y\left(\theta ; x_{0}\right)\right)=-\left.\theta \frac{d v\left(y\left(\theta ; x_{0}\right)\right)}{d t}\right|_{(4.1)}+\left.\frac{1}{2} \frac{d^{2} v\left(y\left(c ; x_{0}\right)\right)}{d t^{2}}\right|_{(4.1)} \theta^{2},
$$

where $c \in(0, \theta)$.

We have

$$
\begin{gathered}
\left.\frac{d v\left(y\left(\theta ; x_{0}\right)\right)}{d t}\right|_{(4.1)}=-\psi\left(x_{0}\right) y^{T}\left(\theta ; x_{0}\right) Q y\left(\theta ; x_{0}\right), \\
\left.\frac{d^{2} v\left(y\left(c ; x_{0}\right)\right)}{d t^{2}}\right|_{(4.1)}=\psi^{2}\left(x_{0}\right) y^{T}\left(c ; x_{0}\right)\left(A_{0}^{T 2} X+2 A_{0}^{T} X A_{0}+X A_{0}^{2}\right) y\left(c ; x_{0}\right) \geq 0,
\end{gathered}
$$

where $Q=-\left(A_{0}^{T} X+X A_{0}\right)$.

Consequently, we obtain

$$
\begin{gathered}
v\left(y\left(\theta ; x_{0}\right)\right)-v\left(x_{0}\right) \leq-\theta \bar{\psi} y^{T}\left(\theta ; x_{0}\right) Q y\left(\theta ; x_{0}\right)+\theta \frac{\psi_{M}-\psi_{m}}{2}\|Q\|\left\|y\left(\theta ; x_{0}\right)\right\|^{2}, \\
\begin{array}{c}
v\left(y\left(\theta+0 ; x_{0}\right)\right)-v\left(y\left(\theta ; x_{0}\right)\right) \leq \bar{\psi} y^{T}\left(\theta ; x_{0}\right)\left(B^{T} X+X B+\bar{\psi} B^{T} X B\right) y\left(\theta ; x_{0}\right) \\
+\vartheta v\left(y\left(\theta ; x_{0}\right)\right) .
\end{array}
\end{gathered}
$$

This implies that

$$
\begin{aligned}
v\left(y\left(\theta+0 ; x_{0}\right)\right)-v\left(x_{0}\right) & \leq-\bar{\psi} y^{T}\left(\theta ; x_{0}\right) G y\left(\theta ; x_{0}\right)+\vartheta_{1} v\left(y\left(\theta ; x_{0}\right)\right) \\
& \leq\left(-\frac{\bar{\psi} \lambda_{m}(G)}{\lambda_{M}(X)}+\vartheta_{1}\right) v\left(y\left(\theta ; x_{0}\right)\right)
\end{aligned}
$$

where $\vartheta_{1}=\vartheta+\frac{\left(\psi_{M}-\psi_{m}\right)\|Q\| \theta}{2 \lambda_{m}(X)}$.

Let $\delta=\frac{\bar{\psi} \lambda_{m}(G)}{\lambda_{M}(X)}-\vartheta_{1}>0$. Then

$$
\left.\frac{d v}{d t}\right|_{(4.1)}=-\psi\left(x_{0}\right) y^{T} Q y \geq-\psi\left(x_{0}\right) \lambda_{M}(Q)\|y\|^{2} \geq-\Lambda^{*}(Q, X) v(y),
$$


where

$$
\Lambda^{*}(Q, X)=\left\{\begin{array}{lll}
\frac{\lambda_{M}(Q) \psi_{M}}{\lambda_{m}(X)}, & \text { for } & \lambda_{M}(Q)>0 \\
\frac{\lambda_{M}(Q) \psi_{m}}{\lambda_{M}(X)}, & \text { for } & \lambda_{M}(Q) \leq 0
\end{array}\right.
$$

Therefore, we obtain

$$
v\left(y\left(\theta ; x_{0}\right)\right) \geq v\left(x_{0}\right) e^{-\theta \Lambda^{*}(Q, X)} .
$$

Thus, we get

$$
v\left(y\left(\theta+0 ; x_{0}\right)\right)-v\left(x_{0}\right) \leq-\delta v\left(y\left(\theta ; x_{0}\right)\right) \leq-\delta e^{-\theta \Lambda^{*}(Q, X)} v\left(x_{0}\right) .
$$

From this it follows the estimate

$$
\left\|U\left(x_{0}\right)\right\| \leq \sqrt{\frac{\lambda_{M}(X)}{\lambda_{m}(X)}\left(1-\delta e^{-\theta \Lambda^{*}(Q, X)}\right)}:=\widetilde{\zeta}_{1} .
$$

Similarly, we obtain

$$
\left\|\left(I+\hat{B}\left(x_{0}\right)\right) e^{\psi\left(x_{0}\right) \theta A_{0}}\right\| \leq(1+\eta) \sqrt{\frac{\lambda_{M}(X)}{\lambda_{m}(X)}} e^{-\psi^{* *} \theta \Lambda(Q, X) / 2}:=\widetilde{\zeta}_{2},
$$

where

$$
\psi^{* *}= \begin{cases}\psi_{M}, & \text { for } \Lambda(Q, X)<0, \\ \psi_{m}, & \text { for } \quad \Lambda(Q, X) \geq 0\end{cases}
$$

The constants $\zeta_{3}, \zeta_{4}, \zeta_{5}$ are determined by the formulas (4.8), and the matrix $R$ by the formula

$$
R=-\left(B^{T} X+X B+\bar{\psi} B^{T} X B\right) .
$$

An immediate consequence of Theorem 1 is the global stability conditions for the solution $x=0$ of the system of nonlinear impulsive differential equations (4.1).

Proposition 2. Assume that for system (4.1) the conditions (4.2) are fulfilled and for a given symmetric positive-definite matrix $G$ there exists a symmetric positivedefinite matrix $X$ satisfying conditions (4.9). If

$$
\frac{\bar{\psi} \lambda_{m}(G)}{\lambda_{M}(X)}-\vartheta_{1}>0, \quad \widetilde{\zeta}_{1}+\zeta_{4} \zeta_{3}+\zeta_{5} \widetilde{\zeta}_{2}+\zeta_{4} \zeta_{5}<1
$$

then the solution $x=0$ of system (4.1) is globally asymptotically stable.

\section{NUMERICAL EXAMPLE}

Let us consider the linear impulsive system (4.1) with matrices

$$
A(t)=A_{0}+A_{1} \cos \omega t+A_{2} \sin \omega t, \quad B=\left(\begin{array}{cc}
-0.5 & 0 \\
0 & 0.25
\end{array}\right),
$$


where $\omega=\frac{2 \pi}{\theta}$ and

$$
A_{0}=\left(\begin{array}{cc}
1 & 0 \\
0 & -1
\end{array}\right), \quad A_{1}=\left(\begin{array}{ll}
0 & 1 \\
0 & 0
\end{array}\right), \quad A_{2}=\left(\begin{array}{ll}
0 & 0 \\
1 & 0
\end{array}\right) .
$$

Let $\psi_{M}=0.205, \psi_{m}=0.195, \sigma=0.004, \theta=0.365625$. Next we will study the asymptotic stability of considered system based on Proposition 2. Let $X=I$. Then we obtain $\eta \leq 0.01027, \eta_{0} \leq 0.00551, \eta_{1} \leq 1.10801, \vartheta_{1} \leq 0.03203, \lambda_{m}(G)=$ $0.21875, \delta \geq 0.01172, \widetilde{\zeta}_{1} \leq 0.99495, \zeta_{3} \leq 1.0525, \zeta_{4} \leq 0.00193, \zeta_{5} \leq 0.00180$.

Hence, if the inequality $\widetilde{\zeta}_{1}+\zeta_{4} \zeta_{3}+\zeta_{5} \widetilde{\zeta}_{2}+\zeta_{4} \zeta_{5}<1, \delta>0$ is fulfilled, then the hypotheses of Proposition 2 are satisfied and the linear impulsive system (4.1) is asymptotically stable for the particular case.

We note that the matrix $A_{0}$ does not satisfy the Routh-Hurwitz conditions, and also the condition $r_{\sigma}(I+B)<1$ does not hold for the matrix $B$. Therefore, the construction of the Lyapunov function for system (4.1) in this particular case is difficult.

\section{DISCUSSION OF RESULTS}

First of all, we note that the problem of global asymptotic stability of the zero solution for a nonlinear impulsive differential equation has some specificity in comparison with a similar problem for differential equations without impulsive action and it is more complicated. For example, if we consider the linear system of differential equations (4.1) in the case when there is no impulsive action, i.e. $B=0$, then the solution $x=0$ of the system (4.1) will be globally asymptotically stable if $\psi(x)>0$.

If $B \neq 0$, then from the asymptotic stability of the linear system

$$
\begin{aligned}
& \frac{d x(t)}{d t}=A(t) x(t), \quad t \neq n \theta, \\
& \Delta x(t)=B x(t), \quad t=n \theta,
\end{aligned}
$$

generally speaking, does not follow the global asymptotic stability of the equilibrium position $x=0$ of the system (4.1). Let us consider a one-dimensional impulsive differential equation

$$
\begin{aligned}
& \frac{d x(t)}{d t}=x(t), \quad t \neq n \theta, \\
& \Delta x(t)=-\gamma x(t), \quad t=n \theta,
\end{aligned}
$$

where $x \in \mathbb{R}, \gamma \in(0,1)$.

Obviously, the inequality $e^{\theta}(1-\gamma)<1$ guarantees (global) asymptotic stability of the linear impulsive differential equation (6.1). Let us consider a nonlinear impulsive differential equation

$$
\begin{aligned}
& \frac{d x(t)}{d t}=x(t)\left(1+x^{2}(t)\right), \quad t \neq n \theta, \\
& \Delta x(t)=-\gamma x(t)\left(1+x^{2}(t)\right), \quad t=n \theta .
\end{aligned}
$$


The zero solution of this equation is locally asymptotically stable (under the condition $\left.e^{\theta}(1-\gamma)<1\right)$. Let us prove that the solution $x=0$ is not globally asymptotically stable.

Let $x\left(t, x_{0}\right)$ be a solution of the Cauchy problem (6.2), $x\left(0+, x_{0}\right)=x_{0}, \omega^{+}\left(x_{0}\right)$ is a length of the right maximal interval of existence of the solution of the corresponding ordinary differential equation without impulsive action. It is easy to see that $\omega^{+}\left(x_{0}\right)<\frac{1}{2 x_{0}^{2}}$. Therefore, for all $x_{0}>\frac{1}{\sqrt{\theta}}$, the inequality $\omega^{+}\left(x_{0}\right)<\frac{\theta}{2}$ is fulfilled. Hence, such a solution $x\left(t, x_{0}\right)$ goes to infinity until the moment of the first impulse action. Therefore, this solution is not infinitely-prolongable, and, as a result, the solution $x=0$ is not globally asymptotically stable.

The proposed method of investigating the global asymptotic stability reduces the problem of stability of the solution of the linear operator equations and makes it possible to establish the global asymptotic stability under various assumptions about the dynamic properties of the continuous and discrete components of the impulsive system. The idea of applying the methods of commutator calculus in problems of stability theory of the nonlinear impulsive equations in a Banach space is new and opens up new possibilities for the development of the Lyapunov's direct method for the stability study of solutions of nonautonomous differential equations.

\section{ACKNOWLEDGEMENT}

This research was completed with the support of the Scientific and Technological Research Council of Turkey (2221-Fellowships for Visiting Scientists and Scientists on Sabbatical Leave 2221-2017/2 period) when Vitalii Slynko was a visiting scholar at Van Yuzuncu Yil University, Van, Turkey.

\section{REFERENCES}

[1] J. L. Dalec'kii and M. G. Krein, Stability of solutions of differential equations in Banach space. Translated from the Russian by S. Smith. Translations of Mathematical Monographs, Vol. 43. American Mathematical Society, Providence, R.I., 1974.

[2] A. Dvirnyi and V. Slyn'ko, "Global stability of solutions of nonstationary monotone differential equations with impulsive action in the pseudolinear form." Nonlinear Oscil., N.Y., vol. 14, no. 2, pp. 193-210, 2011, doi: 10.1007/s11072-011-0151-3.

[3] A. Dvirnyi and V. Slyn'ko, "Application of Lyapunov's direct method to the study of the stability of solutions to systems of impulsive differential equations." Math. Notes, vol. 96, no. 1, pp. 26-37, 2014, doi: 10.1134/S0001434614070037.

[4] A. Ignat'ev, O. Ignat'ev, and A. Soliman, "Asymptotic stability and instability of the solutions of systems with impulse action." Math. Notes, vol. 80, no. 4, pp. 491-499, 2006, doi: 10.1007/s11006-006-0167-7.

[5] K. Ireland and M. Rosen, A classical introduction to modern number theory. 2nd ed., 2nd ed. New York etc.: Springer-Verlag, 1990.

[6] L. V. Kantorovich and G. P. Akilov, Functional analysis., 2nd ed. Pergamon Press, OxfordElmsford, N.Y., 1982. 
[7] V. Lakshmikantham, D. Bainov, and P. Simeonov, Theory of impulsive differential equations. Series in Modern Applied Mathematics, v. 6. New Jersey: World Scientific Publishing Co., Inc., Teaneck, 1989.

[8] X. Liu and A. Willms, "Stability analysis and applications to large scale impulsive systems: A new approach." Can. Appl. Math. Q., vol. 3, no. 4, pp. 419-444, 1995.

[9] W. Magnus, "On the exponential solution of differential equations for a linear operator." Commun. Pure Appl. Math., vol. 7, pp. 649-673, 1954, doi: 10.1002/cpa.3160070404.

[10] R. Medina and M. Gil', "The freezing method for abstract nonlinear difference equations." $J$. Math. Anal. Appl., vol. 330, no. 1, pp. 195-206, 2007, doi: 10.1016/j.jmaa.2006.07.074.

[11] A. Samoilenko and N. A. Perestyuk, Impulsive differential equations. New Jersey: World Scientific Publishing Co., Inc., River Edge, 1995.

[12] V. Y. Slyusarchuk, Instability of Solutions of Evolution Equations. Nat. Univ. of Water Econ. and Nature Manag., Rivne, 2004.

[13] C. Tunc and T. Ayhan, "Global existence and boundedness on a certain nonlinear integrodifferential equation of second order." Dyn. Contin. Discrete Impuls. Syst., Ser. A, Math. Anal., vol. 24, no. 1, pp. 69-77, 2017.

\section{Authors' addresses}

\section{V.I. Slyn'ko}

S.P. Timoshenko Institute of Mechanics of NAS of Ukraine, Stability of Processes Department, 3 Nesterov st., 03680, MSP, Kiev 57, Ukraine

E-mail address: vitstaboukr.net

\section{Cemil Tunç}

Van Yuzuncu Yil University, Department of Mathematics, Faculty of Sciences, 65080-Kampus, Van, Turkey

E-mail address: cemtunc@yahoo.com 